\title{
APROXIMAÇÕES ENTRE NEUROCIÊNCIAS E EDUCAÇÃO: uma revisão sistemática
}

\author{
Hercio da Silva Ferreira ${ }^{l}$ \\ Tadeu Oliver Gonçalves² \\ Soraia Valéria de Oliveira Coelho Lameirão 3
}

\section{RESUMO}

A chamada 'década do cérebro' se caracterizou pelos avanços nas Ciências Neurológicas e isso despertou nos cientistas, um grande entusiasmo no estudo do funcionamento cerebral. Tanto, que alguns educadores viram a possibilidade de aplicar resultados das Neurociências, diretamente em sala de aula. A partir daí, surge uma profunda discussão no meio científico, sobre a possibilidade e/ou necessidade de aplicação desses resultados diretamente em sala de aula e sobre quem está habilitado a fazer tal aplicação. Dessas discussões, surgiram várias iniciativas de fazer emergir um novo campo de pesquisa, capaz de estudar cientificamente a melhor forma de aproximar as Neurociências da Educação. Neste artigo, discutimos como a 'década do cérebro' influenciou o surgimento deste novo campo de pesquisa - que alguns chamam 'Neuroeducação' e outros, 'Ciência da Mente, Cérebro e Educação' -, a pesquisa colaborativa entre pesquisadores de áreas diferentes, levando em consideração a multidisciplinaridade, a interdisciplinaridade e a transdisciplinaridade e os modelos de pesquisa colaborativa, propostos para este novo campo. Discutimos também quão complexa é a análise das dificuldades de aprendizagem em leitura/escrita e em matemática, dando ênfase ao 'Modelo de Níveis de Desenvolvimento' proposto por Jodi Tommerdahl.

Palavras-chave: Neuroeducação. Transdisciplinaridade. Ensino-aprendizagem.

\footnotetext{
1 Doutorando do Programa de Pós-Graduação em Educação em Ciências e Matemáticas (PPGECM/UFPA). Professor adjunto da Universidade Federal Do Pará-UFPA, Instituto de Educação Matemática e Científica-IEMCl, Faculdade de Educação Matemática e Científica-FEMCl, Belém, Pará. ORCID iD: https://orcid.org/0000-0003-3417-0174. E-mail: hercio@ufpa.br

2 Doutor em Educação Matemática. Professor Titular da Universidade Federal do Pará-UFPA, Instituto de Educação Matemática e Científica-IEMCl, Faculdade de Educação Matemática e Científica-FEMCl. Docente/Pesquisador do Programa de Pós-Graduação em Educação em Ciências e Matemática (PPGECM/UFPA). Belém, Pará. ORCID iD: https://orcid.org/00000002-2704-5853. E-mail: tadeuoliver@yahoo.com.br

3 Doutora em Neurociências e Biologia Celular. Professor Associado da Universidade Federal do Pará-UFPA, Instituto de Educação Matemática e Científica-IEMCl, Faculdade de Educação Matemática e Científica-FEMCl. Belém, Pará. ORCID iD: https://orcid.org/00000001-8449-3823. E-mail: soraialameirao@gmail.com
} 


\title{
APPROACHES BETWEEN NEUROSCIENCES AND EDUCATION: a systematic
}

\author{
review
}

\begin{abstract}
The so-called 'decade of the brain' was characterized by the advances in Neurosciences which aw akened great enthusiasm in the study of brain functioning in the scientific world, so much so that some educators saw the possibility of introducing Neuroscience results directly into the classroom. Thenceforth, a deep discussion arises in the scientific environment about the possibility and/or necessity of introducing these results directly into the classroom and about who is qualified to make such introduction. From these discussions have emerged several initiatives to establish a new field of research capable of scientifically studying the best way to relate Neurosciences to Education. In this article, it is discussed how the 'decade of the brain' has influenced the emergence of this new field of research, which some call 'Neuroeducation' and others, 'Mind, Brain and Education', the collaborative research among researchers from different areas, taking into account the multidisciplinarity, interdisciplinarity and transdisciplinarity and the models of collaborative research proposed for this new field. We also discuss the complexity of the analysis of learning difficulties in reading / writing and mathematics, with emphasis on the Model of Development Levels proposed by Jodi Tommerdahl.
\end{abstract}

Keywords: Neuroeducation. Transdisciplinarity. Teaching-learning.

\section{APROXIMACIONES ENTRE NEUROCIENCIAS Y EDUCACIÓN: Una revisión}

\section{sistemática}

\section{RESUMEN}

La llamada 'década del cerebro' se caracterizó por los avances en las Ciencias Neurológicas y eso despertó en los científicos un gran entusiasmo en el estudio del funcionamiento cerebral. Tanto que algunos educadores miraron la posibilidad de aplicar resultados de las Neurociencias directamente en el aula. A partir de ahí surge una profunda discusión en el medio científico sobre la posibilidad y / o necesidad de aplicación de esos resultados directamente en el aula y sobre quién está habilitado a hacer tal aplicación. De estas discusiones surgieron varias iniciativ as de hacer emerger un nuevo campo de investigación capaz de estudiar científicamente la mejor forma de aproximar las Neurociencias de la Educación. En este artículo discutimos cómo la 'década del cerebro' influenció el surgimiento de este nuevo campo de investigación -que algunos llaman 'Neuroeducación' y otros, 'Ciencia de la mente, cerebro y educación' -, la investigación colaborativa entre investigadores de áreas diferentes, llevando en la consideración de la multidisciplinariedad, la interdisciplinariedad y la transdisciplinariedad y los modelos de investigación colaborativa propuestos para este nuevo campo. También discutimos cuán compleja es el análisis de las dificultades de aprendizaje en lectura / escritura y en matemáticas, dando énfasis al "Modelo de Niveles de Desarrollo" propuesto por Jodi Tommerdahl.

Palabras clave: Neuroeducación. Transdisciplinario. Enseñanza y aprendizaje. 


\section{INTRODUÇÃO}

No final dos anos 80 surgem nos Estados Unidos, a partir de um grupo de pesquisadores da época, discussões a respeito das possibilidades de avanço das Ciências Neurológicas, tanto no ensino básico, quanto no clínico. O resultado dessas questões deu origem em 1988, à proposta de que, os anos 90 seriam conhecidos como a 'Década do Cérebro', quando, em um esforço nacional, diferentes cientistas se dedicariam a entender melhor, como o cérebro (e o sistema nervoso) estaria organizado, procurando, não somente, conhecer o funcionamento de um cérebro sadio, mas também, de um cérebro deficiente (GOLDSTEIN, 1994).

Assim, a partir de um esforço coletivo de diferentes políticos, no Congresso e no Senado norte-americano, em 25 de julho de 1989, o presidente Bush assinou um decreto presidencial, conclamando os Estados Unidos a participarem da 'Década do Cérebro'. Embora, inicialmente, este movimento tenha sofrido com limitações financeiras, as pesquisas avançaram e a proposta do presidente foi considerada um sucesso científico. Atualmente, as Ciências Neurológicas, Básica e Clínica são identificadas como um importante componente da agenda nacional de pesquisa e cerca de US $\$ 1,5$ bilhões é dedicado à pesquisa sobre o sistema nervoso, principalmente, através dos programas do Instituto Nacional de Saúde (NIH) (GOLDSTEIN, 1994).

A partir dos Estados Unidos, iniciou-se uma reação em cadeia ao redor do mundo e várias federações e sociedades adotaram a 'Década do 'Cérebro', o que representou, segundo algumas autoridades, um avanço de mais de 50 anos, em pesquisas sobre o sistema nervoso. A 'Década do Cérebro' se propagou, simultaneamente, pelo Reino Unido e Europa e permitiu o desenvolvimento de técnicas e tecnologias para o estudo do cérebro humano intacto, promovendo o crescimento das pesquisas em Neurociências e favorecendo o nascimento de algumas disciplinas como: neurobiologia, neurogenética, neurociência computacional, neuroinformática, neurociência cognitiva e transplantes neurais (TANDON, 2000). 
Antes da 'década do cérebro', mapear regiões do cérebro humano pelo seu papel funcional, parecia uma tarefa muito longe de ser alcançada, mas, com os enormes avanços da Neurociência nos últimos anos, isso já é uma realidade. Na verdade, em plena década do cérebro, Pechura e Martin (1991) observavam essas mudanças no mapeamento cerebral, com o surgimento de novos recursos para o estudo das estruturas e funções cerebrais. Nesta época, a grande expectativa, girava em torno dos benefícios que estes novos recursos de pesquisa trariam para os distúrbios ment ais e neurológicos:

O desafio agora é estabelecer uma iniciativa abrangente que aumente a capacidade dos neurocientistas de fazer descobertas sobre o cérebro e aplicar esse conhecimento aos muitos distúrbios mentais e neurológicos que afetam a humanidade (PECHURA; MARTIN, 1991, p.25).

Portanto, a 'Década do Cérebro' ficou marcada pelos significativos resultados baseados em neuroimagens, os quais causaram grande entusiasmo à comunidade científica, mas também, levaram alguns educadores a interpretações precipitadas e generalizadas. O principal questionamento que ficou desta década, gira em torno de como a Neurociência pode subsidiar a Educação, de forma a melhorar o ensinoaprendizagem em sala de aula. Este questionamento vem sendo discutido até os dias de hoje e veremos os avanços e os desafios que surgiram dessas discussões.

\section{O MODELO DE BRUER}

Foi no final da década do cérebro que John T. Brver despertou o interesse da comunidade científica para o que ele chamou de 'Argumento da Neurociência e Educação'. Bruer (1997) conta que, na década de 1990 , muitos educadores demonstraram um grande entusiasmo, por meio de publicações, em como a compreensão emergente do desenvolvimento cerebral e das funções neurais poderia revolucionar a prática educacional. O 'argumento da Neurociência e Educação', ao qual Bruer (1997) se refere, 
baseia-se em três descobertas da Neurobiologia do Desenvolvimento que levaram a interpretações e conclusões precipitadas, da parte de educadores, em querer aplicar essas descobertas diretamente em sala de aula. Primeiro, os neurocientistas descobriram que durante a infância ocorre um aumento dramático no número de sinapses (sinaptogênese) e em seguida, ocorre um período de eliminação sináptica (poda sináptica). Segundo, existem Períodos Críticos que dependem de experiências no desenvolvimento de sistemas sensoriais e motores. Terceiro, em ratos, pelo menos, ambientes complexos ou enriquecidos fazem com que novas sinapses se formem (BRUER, 1997, p. 4).

Segundo Bruer (1997), devemos ser cautelosos ao interpretar e tirar conclusões dessas três descobertas, para aplicar na educação, por exemplo, não se sabe exatamente qual a relação da sinaptogênese com a aquisição de habilidades de leitura e aritmética que as crianças adquirem por meio de interação social informal e instrução escolar formal. Por isso, advertiu que, a aplicação desses resultados, diretamente na educação seria uma ponte longa demais. Brver (1997) acreditava baseado nas descobertas da época, que o melhor caminho para aplicar os resultados da Neurociência na Educação seria utilizar duas pontes mais curtas (Fig.1):

Há uma ponte bem estabelecida, hoje com quase 50 anos, entre Educação e Psicologia Cognitiva. Há uma segunda ponte, com apenas 10 anos de idade, entre a Psicologia Cognitiva e a Neurociência (BRUER, 1997, p. 4).

FIGURA 1. As duas pontes de Bruer

\begin{tabular}{|c|c|c|c|c|}
\hline $\begin{array}{l}\text { Prática } \\
\text { Educacional }\end{array}$ & $\begin{array}{l}19 \\
\text { ponte }\end{array}$ & $\begin{array}{l}\text { Psicologia } \\
\text { Cognitiva }\end{array}$ & ponte & $\begin{array}{l}\text { Neurociencia } \\
\text { Cognitiva }\end{array}$ \\
\hline
\end{tabular}

Goswami (2006) vai além e classifica a aplicação da sinaptogênese e de períodos críticos nas escolas, como neuromitos ${ }^{4}$ que precisam ser

\footnotetext{
${ }^{4}$ Neuromito: equívoco gerado por um mal-entendido, uma leitura errada ou uma citação errônea de fatos cientificamente estabelecidos (por pesquisa do cérebro) para justificar o
} 
combatidos para que os verdadeiros avanços da Neurociência Cognitiva possam ser revelados. Há quem afirme que o problema dos neuromitos nas escolas, poderia ser combatido, se a neurociência fosse incluída como disciplina obrigatória na formação dos professores. Howard-Jones (2014) afirma que "atualmente, os professores estão mal preparados para criticar ideias e programas educacionais que reivindicam uma base neurocientífica". Para Ansari (2015, p.1710):

os programas de formação de professores deveriam treinar sistematicamente os professores na avaliação do conhecimento empírico durante o 'Pre-service Training', então esses indivíduos estariam mais bem equipados para usar evidências para subsidiar suas decisões pedagógicas.

E o problema pode ainda ser maior, se considerarmos a possibilidade de os neuromitos inibirem a aprendizagem do aluno, em vez de melhorá-la (veja: Tommerdahl 2008; Ansari 2015).

Alguns dos neuromitos mais populares, segundo Rato, J. e Caldas, A. (2010) são: O uso de apenas $10 \%$ do cérebro; o funcionamento cerebral esquerdo e direito, como independentes; as múltiplas inteligências; os estilos de aprendizagem baseados nas pedagogias multissensoriais; 0 beber bastante água para melhorar a aprendizagem.

Evidentemente, que se trata de uma problemática ainda muito discutida nos dias de hoje, pois, quando analisamos o sujeito dentro de um ambiente escolar, muitas outras variáveis devem ser levadas em consideração e com certeza, essa era uma das preocupações de Bruer (1997), ao analisar a forma como educadores interpretavam as descobertas da Neurociência, sem levar em consideração, o ambiente e as condições envolvidas nessas descobertas. Entretanto, Bruer (1997) admite que a plasticidade cerebral pode fornecer uma base neural para a aprendizagem formal e informal que ocorre nos ambientes socioculturais, inclusive nas escolas, mas é preciso cautela nas interpretações. Enfim, todas essas

uso da pesquisa do cérebro na educação e em outros contextos. Fonte: OECD Publications, 2002. 
descobertas da Neurociência e as possibilidades de aplicação na educação necessitavam, segundo Bruer (1997), de mais amadurecimento e muita cautela e tentar aplicar essas descobertas diretamente na educação era uma ponte longa demais.

Segundo Bruer (1997), é necessário que a Psicologia Cognitiva forneça os seus resultados de pesquisas comportamentais da prática escolar $\left(1^{a}\right.$ ponte) à Neurociência Cognitiva, para que esta possa pesquisar que relações existem entre esses resultados e as atividades cerebrais ( $2^{a}$ ponte). Desta forma, é possível inferir como o estudo do cérebro pode ajudar nas práticas educacionais. Como exemplo da primeira ponte, Psicólogos Cognitivos descobriram que alunos iniciantes na escola, que não trazem habilidade em comparação numérica - Qual número é maior, 5 ou 4? -, terão dificuldades na instrução de aritmética formal na escola. Essa habilidade de comparação numérica, as crianças adquirem informalmente com seus irmãos mais velhos, pais, enfim, com seus familiares. Pesquisas apontaram que crianças de origem nas classes mais baixas da sociedade dos Estados Unidos, não trazem essas habilidades de casa ao iniciarem a vida escolar, e que esse é um grande problema que precisa ser superado no início das atividades escolares formais, no sentido de nivelar esses alunos com os demais das classes média e alta. Como um exemplo da $2^{a}$ ponte, Neurocientistas Cognitivos buscam entender como as estruturas neurais e os circuitos cerebrais implementam os processos cognitivos em habilidades como a comparação numérica. A partir de tecnologias de imagem e gravação do cérebro de população de aprendizado normal, poderemos entender melhor as necessidades educacionais de populações especiais (BRUER, 1997, p.13).

Kurt Fischer parece não concordar com Bruer (1997), no que diz respeito a levar resultados biológicos diretamente para a educação. Segundo Fischer (2009), os exemplos usados por Bruer (1997) são limitados e omitem a grande utilidade da análise biológica, na promoção de objetivos educacionais. Além disso, segundo Fischer (2009), Bruer (1997) desconsidera a utilidade de levar conceitos biológicos diretamente às práticas 
educacionais, para se pensar sobre muitas situações escolares. Como contraexemplo, Fischer (2009) usa o caso das crianças com epilepsia severa que tiveram de passar por procedimento cirúrgico, para a remoção de um hemisfério do cérebro e por isso são chamadas 'crianças de meio-cérebro'. O fato é que, ao contrário das expectativas, algumas das crianças de meiocérebro cresceram em ambientes que são altamente favoráveis à aprendizagem e desenvolveram fortes habilidades - até mesmo, habilidades que a neurociência tradicional, indica que elas não deveriam ser capazes de desenvolver. Isto implica que "educar uma 'criança de meio-cérebro' requer conhecimento da biologia do cérebro e do corpo, especialmente dos problemas especiais que são criados pela perda de um hemisfério" (FISCHER, 2009, p.6). Neste caso, as descobertas da Neurociência devem chegar diretamente a todos os envolvidos na educação destas crianças. Outros trabalhos contribuem nesta discussão, reforçando a proposição de que é possível, e em alguns casos, necessário, que a Neurociência aplique suas descobertas diretamente à Educação. Por exemplo, resultados recentes da Neurociência, através de medidas de neuroimagem, mostram que é possível prever se uma criança terá ou não dificuldades em leitura e/ou matemática (veja: Ansari, 2015).

O fato é que a preocupação de Bruer (1997) com o grande entusiasmo, de querer levar resultados da Neurociência, diretamente para a sala de aula, era, em parte, pertinente e parecia prever a atuação de pessoas desqualificadas, intermediando o diálogo direto entre a Neurociência e a escola. Com relação a isto, Fischer (2009) concorda e também demonstra muita preocupação com o que ele chama de 'tentativas irresponsáveis' de venda de muitos projetos comerciais com reinvindicações que são 'baseadas no cérebro'. A simples tentativa de generalização das descobertas das Neurociências, sem a devida preocupação científica e ética, pode levar a atitudes precipitadas e inconsequentes. Por exemplo, "estudos sugerem que a aplicação de corrente elétrica fraca ao cérebro através de eletrodos no couro cabeludo pode levar a melhorias das funções cognitivas" (ANSARI, 2015, p. 1714), 
porém, a amostra desta pesquisa, além de pequena, era composta de indivíduos adultos. Generalizar esta pesquisa, sugerindo a aplicação deste método em crianças, pode levar a atitudes precipitadas e inconsequentes.

Também Goswami (2006) demonstra toda a sua preocupação, em fazer algo que possa frear a aplicação errônea da Neurociência à Educação e fala isso, baseado no que ela chama de 'indústria de aprendizagem baseada no cérebro' e que o atual abismo entre a Neurociência e a Educação, está sendo preenchido por pacotes e programas que se dizem baseados na ciência do cérebro. Na verdade, Goswami (2006) está mais preocupada em uma maneira de informar as descobertas da Neurociência diretamente aos professores:

Podemos aterrar o abismo entre a neurociência e a educação falando diretamente com os professores e evitando os intermediários da indústria da aprendizagem baseada no cérebro? (GOSWAMI, 2006, p.6).

Nesta citação, Usha Goswami se refere aos grandes avanços que foram alcançados pelos neurocientistas cognitivos, no entendimento da dislexia e da discalculia, mas que são resultados que ainda necessitam de desenvolvimento e se preocupa com a possibilidade - e isto é, uma realidade nos dias de hoje - de pessoas não qualificadas levarem esses resultados incipientes à escola. Por isso, a pergunta, se não seria melhor que os próprios cientistas informassem aos professores sobre os avanços alcançados. Quanto a isso, Goswami (2006) nos conta que uma série de seminários foi realizada no Reino Unido, onde cientistas cognitivos informaram diretamente aos professores, os resultados de suas pesquisas. E que na conferência de Cambridge, destacados neurocientistas que trabalham em áreas como alfabetização, numeração, QI, aprendizagem, cognição social e TDAH falaram diretamente com os professores, sobre as evidências científicas que estavam sendo coletadas nos laboratórios dos cientistas. O resultado desses encontros entre cientistas e professores, teve como ponto positivo, o esclarecimento de que muitos dos programas 
baseados no cérebro, aplicados nas escolas, não tinham nenhuma base científica, mas, mostrou também, que neurocientistas não possuem habilidades para falar com professores, uma vez que os cientistas estão muito preocupados em estabelecer o rigor de suas pesquisas experimentais, o que inviabiliza uma compreensão por parte dos professores. Para Goswami (2006), o melhor seria criar uma rede de comunicadores das pesquisas científicas, para que esses pudessem interpretar a neurociência a partir de uma perspectiva e linguagem dos educadores, assim como, no caminho inverso, as teorias educacionais pudessem ser conhecidas pelos neurocientistas.

Parece então que a comunicação - ou a falta de uma comunicação adequada - entre a neurociência e a educação, é um problema sério que precisa ser contornado. Para Howard-Jones (2014), trata-se de um problema que envolve a terminologia e a linguagem diferente, utilizadas por neurocientistas e educadores, e isto pode estar por trás dos processos que levam o conhecimento científico a ser compreendido de forma errônea. No entanto, para Howard-Jones (2014), diferentemente da proposta de Goswami (2006), não há necessidade da formação de comunicadores de pesquisa que atuem na interseção da Neurociência com a Educação. Para que a comunicação seja eficaz, neurocientistas devem trabalhar em colaboração direta com os educadores, que são os profissionais que estão mais familiarizados com as condições culturais e os conceitos da educação.

\section{TRANSDISCIPLINARIDADE}

Uma forma de promover essa colaboração seria aproximar os programas de pesquisa das duas áreas, Neurociência e Educação. Poderíamos criar 'Programas de Colaboração Científica' entre Neurociência e Educação, com o objetivo de possibilitar que pesquisadores dessas duas áreas trabalhem juntos. Este seria um caminho para levar as promissoras pesquisas da Neurociência à sala de aula. Na verdade, a transdisciplinaridade, que une e funde áreas científicas diferentes e leva a um novo campo científico, parece ser o melhor caminho para realizar tal 
façanha. Koizumi (1999) discute essa questão de transdisciplinaridade por meio das figuras $2 a$ e $2 b$ abaixo:

Fig. 2a. Inter ou multidisciplinaridade

Fig. 2b. Transdisciplinaridade
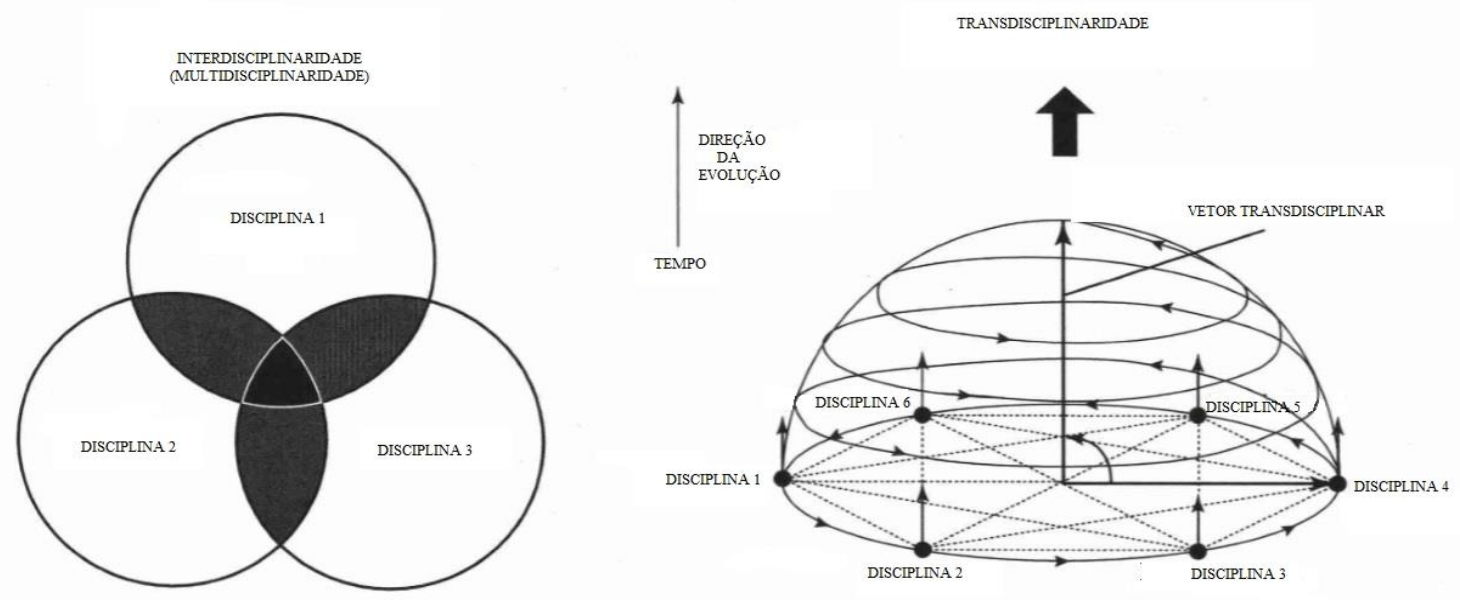

Fonte: Koizumi (1999, p.8)

A multidisciplinaridade está situada num plano bidimensional (Fig. 2a), enquanto a transdisciplinaridade ocupa um espaço tridimensional (Fig. 2b). O conceito de transdisciplinaridade existe em um nível hierárquico superior, produzido pela ligação de várias áreas científicas diferentes no nível hierárquico inferior. Para Koizumi (1999), a transdisciplinaridade inclui os conceitos de ponte e fusão entre áreas completamente diferentes. No entanto, é necessária alguma força motriz para interligar e fundir áreas e impulsionar a evolução de uma nova área abrangente e isso exige novas metodologias e novas organizações de pesquisa, incluindo uma linguagem comum, que possibilitem transcender as fronteiras que separam as disciplinas. Uma simples combinação de múltiplas áreas não é suficiente:

Uma simples combinação de múltiplas áreas não é suficiente para impulsionar $\circ$ vetor transdisciplinar na Fig. $2 b$ na direção perpendicular ao plano multidisciplinar, que é a direção para a qual uma nova disciplina abrangente irá evoluir (KOIZUMl, 1999, p.8).

Em 2009, Boba M. Samuels retoma essa discussão, admitindo que o caminho para uma pesquisa transdisciplinar que possa levar a Neurociência 
ao encontro da Educação, não é uma tarefa simples, mas, possível de ser realizada. Para Samuels (2009), as diferenças entre neurociência e educação são muitas, incluindo diferenças históricas, filosóficas e epistemológicas. Porém:

\begin{abstract}
essas dificuldades podem ser superadas, não por uma perspectiva teórica ou metodológica ou epistemológica comum, mas por uma questão comum na qual todos os participantes transdisciplinares aplicam suas próprias especializações com o objetivo de alcançar uma compreensão holística da questão (SAMUELS, 2009, p.49).
\end{abstract}

Portanto, para Samuels (2009), multidisciplinaridade é a soma de conhecimentos individuais, compartilhados por especialistas ou grupos especializados, enquanto interdisciplinaridade é o conhecimento criado na interseção de disciplinas estabelecidas e que a transdisciplinaridade se caracteriza como um novo tipo de conhecimento, que surge da interação de diversas pessoas dentro de um grupo inteiramente novo (Fig. 3), mas, para que isso seja possível, é necessário derrubar os muros que foram construídos para garantir a soberania de cada campo científico.

FIGURA 3. Multi, Inter e Transdisciplinaridade

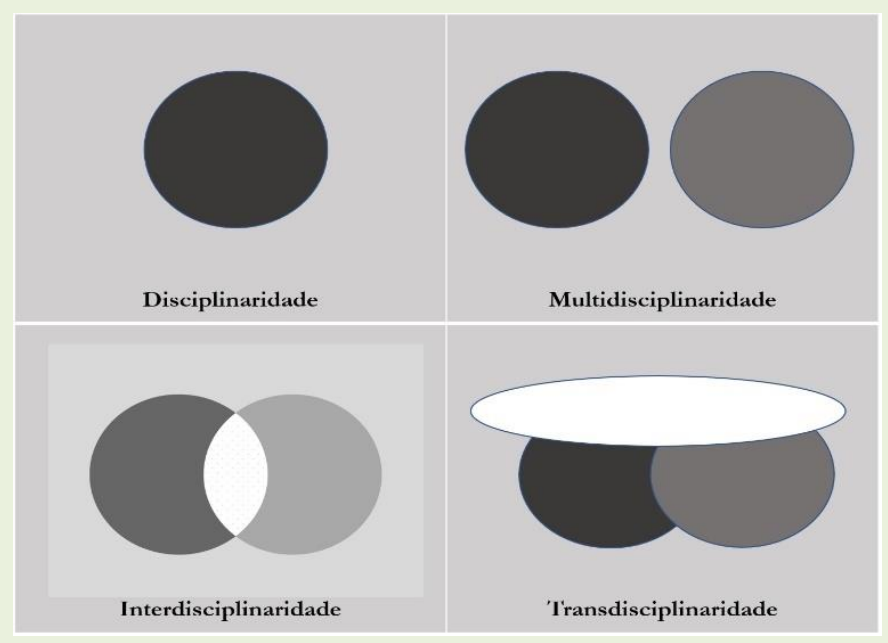

Fonte: Samuels (2009, p.50)

Encontramos em Koizumi (1999), um excelente exemplo de trabalho em equipe multidisciplinar bem-sucedido, realizado pelo casal Pierre e Marie Curie e o químico analítico Gustave Bemont, no final do século 19, que 
interligou e fundiu Matemática, Física e Química e este trabalho inicial abriu caminho para um novo campo científico, a Medicina Nuclear. O próprio Hideaki Koizumi coordenou no Japão, no final do século 20, o 'Instituto Virtual para Estudos Transdisciplinares', que funcionava com auxílio da Internet, e que tinha como objetivo, entre outras coisas, avaliar a eficácia de uma organização estruturada especificamente para a investigação transdisciplinar. Essa é uma excelente forma de fomentar a pesquisa transdisciplinar, visto que dispensa a presença física do pesquisador, atendendo perfeit amente às tendências atuais de pesquisa globalizada.

\section{AS INICIATIVAS DE FAZER EMERGIR UM NOVO CAMPO CIENTÍFICO}

Voltando à década do cérebro, em alguns países, quase simultaneamente, pesquisadores despertaram grande interesse em relacionar a Biologia e Ciência Cognitiva com a Educação. Fischer (2009, p.4) nos conta que:

nos últimos anos do século $X X$, algo borbulhou quase simultaneamente em Paris, Tóquio e Cambridge, Massachusetts - um interesse em trazer a biologia e a ciência cognitiva para um relacionamento próximo com a educação, para promover um conhecimento mais profundo de aprendizado e ensino.

E foi no século 21, precisamente em 2004, que os grupos de Cambridge, Tóquio e Paris, em colaboração, fundaram a Sociedade Internacional Mente, Cérebro e Educação (IMBES) e lançaram a revista Mente, Cérebro e Educação.

Além dessas, no século 21, também surgiram outras iniciativas que buscavam a consolidação de um campo emergente que uns chamam Neuroeducação, outros chamam Ciência da Mente, Cérebro e Educação (MBE Science), ou ainda Neurociência Educacional, o qual propõe reunir de forma colaborativa pesquisadores da Neurociência, da Psicologia e da Educação. Surgiram ações na 'Organização para Cooperação e Desenvolvimento Econômico' (OECD); na 'Pesquisa em Neurociência na Cúpula da Educação', que reuniu neurocientistas, pesquisadores da 
educação, profissionais e formuladores de políticas, e na 'Associação Europeia para a Pesquisa sobre Aprendizagem e Instrução' (EARLI). Todos esses eventos tinham a missão comum de incentivar a pesquisa interdisciplinar colaborativa e informar sobre as promessas e as armadilhas deste campo em desenvolvimento (ANSARI, DE SMEDT, GRABNER, 2012, p.106).

Tokuhama-Espinosa (2008) também apresenta uma iniciativa interessante de pesquisa em que a autora contou com a colaboração de especialistas nas áreas da Neurociência, Psicologia e Educação, com o intuito de definir parâmetros para padrões neuroeducacionais. Após realizar uma revisão da literatura sobre potenciais resultados da Neurociência, para aplicação em sala de aula, a autora desenvolveu uma meta-análise desse material e os especialistas usaram os critérios estabelecidos pela OCDE (Organização para Cooperação e Desenvolvimento Econômico) para chegar a um consenso sobre o uso de informações neuroeducacionais, em ambientes de sala de aula. A própria autora atuou como coordenadora das discussões que estabeleceram esses padrões. No final de sua pesquisa, Tukuhama-Espinosa (2008) propôs 12 Fundamentos, 22 Princípios, e 10 Diretrizes Instrucionais para um novo modelo para o que ela chamou de Neuroeducação.

Dentre todas essas iniciativas, daremos aqui, um destaque maior para a Sociedade Internacional da Mente, Cérebro e Educação (IMBES), por abrir as portas para pesquisadores de várias áreas e de qualquer lugar do Mundo, que pudessem colaborar para o desenvolvimento do Movimento Mente, Cérebro e Educação (MBE). Foi em 1993 que a Universidade de Harvard respondeu às expectativas da chamada década do cérebro, criando uma interfaculdade denominada Mente, Cérebro e Comportamento (MBB). A ideia de aproximar Neurociência e Educação amadureceu e no início dos anos 2000, sob a liderança de Kurt Fischer, o Programa de Mestrado da Escola de Graduação em Educação de Harvard (HGSE) criou uma opção de Mestrado em MBE. E foi, mergulhada neste cenário de grande entusiasmo, que surgiu em 2004, a Sociedade Internacional da Mente, 
Cérebro e Educação (IMBES), como base de apoio ao crescimento do emergente campo MBE, e três anos depois, surge a primeira edição da revista científica Mente, Cérebro e Educação. Para completar essa grande iniciativa, no outono de 2007, a IMBES realizou sua primeira conferência internacional, com 14 países e abordou mais de 25 tópicos (veja: Schwartz, 2015).

\section{OS MODELOS DE PESQUISA PARA O NOVO CAMPO}

A principal missão da IMBES é a pesquisa colaborativa e o grande desafio é encontrar a forma ideal para fomentar esse tipo de pesquisa. As figuras 4a e 4b abaixo são usadas por Schwartz (2015), para esclarecer a natureza dos desafios que a IMBES e o campo da MBE enfrentam. A figura $4 a$ mostra um modelo de relação linear entre as áreas envolvidas. A dificuldade neste modelo, é que, mover-se de um nível hierárquico para o seguinte, gera regras novas para entender as relações entre variáveis. A figura $4 b$ representa um modelo em forma de diagrama de Venn, cuja importância está na convergência representada pela pequena região central, que sugere um foco único para os colaboradores da pesquisa. No entanto, o que não é óbvio é como a pequena população de indivíduos que se encaixa nos três círculos sobrepostos funcionará em conjunto. Além disso, as conversas que emergem em cada uma das três regiões onde apenas duas disciplinas se sobrepõem (Cérebro-Educação, Mente-Educação e CérebroMente) são únicas e muito diferentes do que está se desdobrando no centro do diagrama (SCHWARTZ, 2015, pp.65-67).

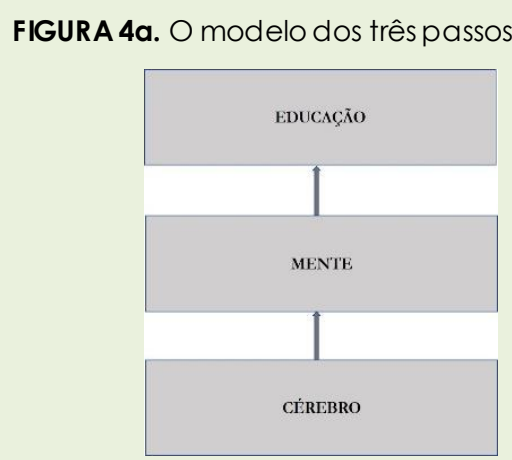

FIGURA 4b. O modelo da sobreposição de disciplinas

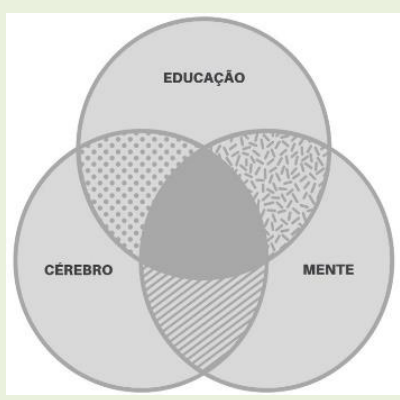

Fonte: (SCHWARTZ, 2015, p.65). 
Mesmo que tenhamos avançado nas pesquisas multidisciplinares e interdisciplinares, muito ainda precisa ser feito para que essas pesquisas alcancem um nível de excelência em que Neurocientistas tenham consciência da importância da pesquisa educacional e das abordagens pedagógicas usadas nas escolas, e no caminho inverso, em que pesquisadores educacionais tenham uma boa percepção das teorias e abordagem metodológica da neurociência. E um dos principais fatores que contribui negativamente, são os muros que a maioria dos pesquisadores insiste em levantar, como uma forma de estabelecer fronteiras que só prejudicam e limitam o desenvolvimento das pesquisas. Uma aproximação mais efetiva entre neurocientistas e educadores, resolveria um grande problema, que surge ao questionarmos qual o impacto dos resultados neurocientíficos de laboratório, quando levados ao contexto escolar. A falta desta compreensão pode levar a deturpações fundamentais de ambos os lados, como os neuromitos, citados anteriormente. Essa questão ecológica é apenas um dos problemas metodológicos a serem superados. O tamanho muito pequeno das amostras, em pesquisas de Neurociência - justificados pelo enorme número de dados coletados durante uma sessão de imagem e pelos custos envolvidos na realização de pesquisas de neuroimagem funcional - em comparação com as amostras das pesquisas educacionais, também é um problema metodológico que precisa ser superado (ANSARI, DE SMEDT, GRABNER, 2012, pp.112-113). Isso reforça ainda mais, as ideias de Koizumi (1999) de criar novas organizações de pesquisa para uma abordagem transdisciplinar.

De fato, até aqui, parece haver um consenso de que mudanças devem acontecer no planejamento das pesquisas que envolvem Neurociência e Educação, para que os resultados possam chegar às salas de aula, onde o ensino ainda não foi efetivamente contemplado pelas descobertas da neurociência. Em relação a essas mudanças, Tommerdahl (2008, p.8) afirma que: 
Apesar do ímpeto crescente desse novo campo da educação, o ensino em sala de aula ainda não foi radicalmente alterado pela introdução de metodologias comprovadas e confiáveis, construídas sobre as ciências do cérebro.

Na verdade, a Neurociência tem avançado na questão do ensinoaprendizagem para crianças com alguma disfunção cognitiva, como a discalculia e a dislexia, mas não tem contribuído no contexto geral de ensino-aprendizagem.

Tommerdahl (2008) discute essa questão de, por que as descobertas das neurociências não estão desempenhando um papel maior nas práticas educacionais, e destaca as três principais dificuldades encontradas: a primeira é que, tanto o estudo do cérebro, realizado por neurocientistas, quanto o estudo do ensino-aprendizagem, realizado por educadores, têm alto grau de complexidade, a segunda são as limitações inerentes aos equipamentos de imagem cerebral e a terceira dificuldade gira em torno do fato de que, as descobertas de laboratórios não podem ser imediatamente, aplicadas à sala de aula, e para esta última dificuldade, a autora propõe um modelo multidisciplinar que discutiremos posteriormente.

Quanto à complexidade, como exemplo, Tommerdahl (2008, p.8) comenta a tarefa de escrever no caderno, uma frase ditada pelo professor em sala de aula:

\footnotetext{
"parece ser uma tarefa simples, mas envolve demandas cognitivas que incluem o processamento linguístico e pragmático da questão, o reconhecimento de palavras na sentença ditada, a transferência das unidades faladas para sinais escritos e a integração de nosso conhecimento mundial sobre como a escrita é realizada, por exemplo, usando uma letra maiúscula no início de uma frase e usando a pontuação e a ortografia corretas. Essa lista poderia se tornar ainda mais complexa, se adicionássemos tarefas como o uso de memória de trabalho e sistemas de atenção".
}

Nessa discussão colocada pela Dr. ${ }^{a}$ Jodi Tommerdahl, ela chama a atenção para o fato de que, analisar as razões pelas quais um determinado aluno não consegue seguir as instruções do professor, no exemplo acima, torna-se muito difícil, as possibilidades são enormes. O fato é que isso não se restringe apenas às questões de Linguagem, o processo de ensinoaprendizagem em Matemática, também tem as suas complexidades. 
Como exemplo de sala de aula, podemos citar uma tarefa de Matemática, comum nas turmas das escolas brasileiras, a partir do $9^{\circ}$ ano, que é resolver uma equação do $2^{\circ}$ grau do tipo $x^{2}+2 x-1=0$, que o aluno poderá resolver aplicando diretamente a Fórmula de Bhaskara $x=\frac{-b \pm \sqrt{b^{2}-4 a c}}{2 a}$ e, se o aluno não lembrar desta fórmula, poderá, por exemplo, resolvê-la através de fatoração. Analisemos agora, a possibilidade de um aluno não conseguir encontrar uma resposta para esta tarefa, que seria, na sua forma mais simplificada, $x=-1+\sqrt{2}$ ou $x=-1-\sqrt{2}$. Se $\circ$ aluno lembrou-se da Fórmula de Bhaskara, mas não consegue realizar os cálculos necessários, então, estamos diante de um problema aritmético que envolve operações de adição, subtração, multiplicação, divisão e radiciação e isso inclui a parte conceitual, as regras de sinal, os algoritmos e as técnicas de resolução. Numa segunda situação, o aluno poderia resolver a tarefa usando fatoração. Neste caso, ele deveria concluir que a equação $x^{2}+2 x-1=0$ é equivalente à equação $(x+1)^{2}=2$ e partir para a solução da segunda equação.

Notemos que descobrir o motivo pelo qual, um aluno não consegue resolver a tarefa discutida acima, é uma análise complexa que envolve muitas variáveis, e que se torna praticamente impossível de analisar, se estivermos fora do contexto da situação. Parece que a sala de aula é o laboratório mais indicado para pesquisar este problema. Quanto a isso, já existem alguns estudos de processos neurais, associados ao processamento matemático que buscam um alto grau de validade ecológica, através de experimentos que se assemelham às tarefas apresentadas em sala de aula, porém, não são realizados no ambiente escolar. Ainda existem outros problemas relatados sobre essa tentativa de alcançar alto grau de validade ecológica: limitações do equipamento de registro de neuroimagem, a amostra era composta só por alunos adultos e, além disso, eram alunos de classe média (veja: Grabner e Ansari, 2010).

Voltando à tarefa matemática proposta acima, poderíamos aqui especular que o aluno não a resolveu porque ele não conseguiu resgatar da 
memória, a fórmula de Bhaskara e nem o método de fatoração. Quanto a isso, propomos uma simulação de como pesquisadores, isoladamente, reagiriam a esta situação.

O Dr. Kurt Fischer afirmaria que devemos ter cautela com modelos que colocam o cérebro como o responsável direto pela aprendizagem de um indivíduo, sem levar em consideração o corpo, os relacionamentos e a cultura de uma pessoa:

Usando esse modelo, as pessoas falam como se o aprendizado ocorresse no cérebro, deixando de fora as formas como o corpo contribui para a aprendizagem, bem como os papéis que o ambiente de uma pessoa desempenha na formação da aprendizagem e no fornecimento de informações (FISCHER, 2009, p. $5)$.

O Dr. Todd Rose diria que para encontrar o motivo pelo qual um determinado aluno não consegue resolver uma tarefa de sala de aula é preciso levar em consideração o comportamento deste aluno em vários contextos:

As respostas às perguntas de "quem" exigem muito mais do que uma compreensão da biologia das diferenças dos alunos com base em medidas dimensionais, como testes de inteligência ou avaliações padronizadas (ROSE; ROSE, 2011, p.155).

O Dr. Daniel Ansari reagiria afirmando que a educação é um processo que induz a plasticidade cerebral, através da instrução em um contexto social e que:

Os professores são os orquestradores da plasticidade neuronal de seus alunos durante o horário de aula. Para que o conhecimento seja adquirido, o cérebro tem que codificar a informação, o que envolve mudanças na conectividade entre as células nervosas (isto é, a plasticidade sináptica) (ANSARI, 2015, p.1704).

O Dr. Alexander Vaninsky diria que pode se tratar de um caso de ansiedade matemática, que na sua opinião é a principal barreira para o sucesso nesta disciplina e por isso deve ser eliminada. Além disso, em alguns casos, nenhuma técnica de ensino funciona: 
tais casos incluem déficit de memória de trabalho que pode resultar em ansiedade matemática, fraca memória de longo prazo que impede 0 armazenamento dos novos fatos por um tempo razoavelmente longo e excessivo ruído de informação nos canais que dificulta a aquisição de informações (VANINSKY, 2017, p.387).

O Dr. Guy Brousseau, provavelmente, faria uso da sua Teoria das Situações Didáticas, para afirmar que esta tarefa deveria fazer parte de uma Situação Didática, em uma referência clara ao Construtivismo de Piaget, em que:

- aluno aprende adaptando-se a um meio que é um fator de contradições, de dificuldades, de desequilíbrios, um pouco como acontece na sociedade humana. Esse saber, fruto da adaptação do aluno, manifesta-se pelas respostas novas, que são a prova da aprendizagem (BROUSSEAU, 1996, p.49).

Não queremos usar essas informações para inferir sobre o problema acima colocado e nem confrontar os teóricos, mas, apenas fazer uma provocação, no sentido de mostrar como são diferentes as formas de olhar um mesmo problema que ocorre em sala de aula e como seria interessante se pesquisadores trabalhassem juntos, na tentativa de melhorar o ensinoaprendizagem, no caso, de matemática.

O fato é que, em ambos os métodos de resolução da tarefa que discutimos acima, precisamos da aritmética para resolver. Portanto, a dificuldade pode estar nas aulas de Matemática dos anos iniciais, uma dificuldade que vem se arrastando ao longo da vida escolar do aluno e que provoca enormes problemas no aprendizado e até nas relações sociais no ambiente escolar. Seja qual for a origem desta dificuldade, em uma pesquisa colaborativa entre as Neurociências, a Psicologia e a Educação Matemática, uma questão de pesquisa, para este problema colocado acima seria "o que está causando a dificuldade em resolver esta tarefa por parte de alguns alunos e quais as estratégias educacionais indicadas para corrigir ou amenizar este problema em sala de aula". A pesquisa poderia iniciar pelos Teóricos da Educação Matemática, através de uma investigação preliminar que envolveria os alunos e o professor da turma - as suas participações seriam imprescindíveis. Em seguida, encaminhada para 
uma discussão multidisciplinar. Esta seria uma forma interessante de levar um problema de sala de aula, para o conhecimento de neurocientistas, psicólogos e educadores e desta forma, fomentar a pesquisa multidisciplinar. Resultados surpreendentes poderiam surgir desta pesquisa colaborativa. Estamos longe de atuarmos conforme os modelos transdisciplinares discutidos acima - Koizumi (1999) e Samuels (2009) - e que parecem ser o melhor caminho para levar a Neurociência ao encontro da Educação, pelo menos até o momento. Portanto, uma pesquisa "multi-inter-disciplinar" já seria um grande avanço, dentro da perspectiva de melhorar o processo ensino-aprendizagem, porque, na verdade, nem isso é feito atualmente. Acreditamos que o conhecimento transdisciplinar surgirá naturalmente, desde que exista o espírito de colaboração, que transcende aos interesses individuais dos participantes desta base de pesquisa.

A segunda dificuldade encontrada por Tommerdahl (2008) são as limitações dos equipamentos de imagem cerebral. Um grande desafio é desenvolver um único equipamento de monitoramento da atividade cerebral, que possua múltiplas capacidades, pois, atualmente, diferentes equipamentos de imagem cerebral têm diferentes capacidades, que permitem diferentes possibilidades. Além disso, alguns equipamentos de monitoramento da atividade cerebral são extremamente barulhentos e outros sofrem interferência com o movimento da cabeça, o que dificulta a participação de crianças.

Em relação à terceira dificuldade encontrada por Tommerdahl (2008), vejamos um modelo de pesquisa multidisciplinar interessante que contempla a discussão acima. Na verdade, a autora propõe um 'tráfego de mão dupla' entre as Teorias Educacionais e as Neurociências, passando pela Psicologia. Inicialmente detectada uma dificuldade na aprendizagem, Teóricos da Educação iniciam a pesquisa e, em seguida, encaminham-na no sentido de cima para baixo, conforme a Fig. 5, até chegar aos Neurocientistas e, no sentido inverso, as descobertas da Neurociência para o problema relat ado devem retornar, passando novamente pela Neurociência Cognitiva, Psicologia, até chegar aos Teóricos da Educação, antes de 
chegar à sala de aula, repetindo este processo, quantas vezes forem necessárias, para se chegar a um consenso do que seria a melhor estratégia educacional, para a questão de pesquisa e, a partir daí, iniciar a Testagem desta estratégia.

FIGURA 5. Modelo de Níveis de Desenvolvimento

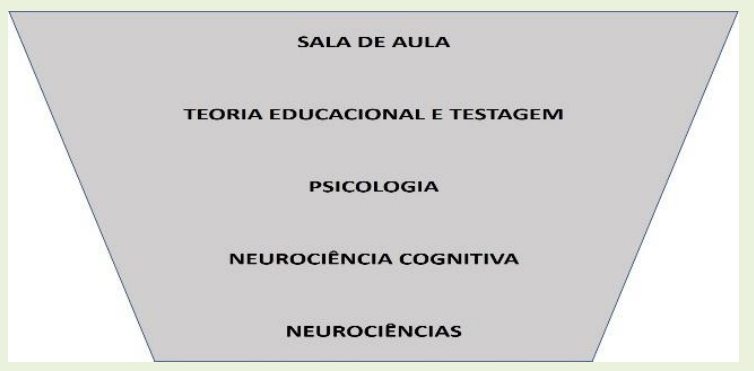

Fonte: Tommerdahl (2008, p.9).

Na verdade, a autora admite que, para o Modelo de Níveis de Desenvolvimento avançar o mais efetivamente possível:

é necessário que os pesquisadores educacionais trabalhem junto com os estudiosos nos níveis cognitivo e psicológico para desenvolver e testar hipóteses sobre o funcionamento dos mecanismos subjacentes à aprendizagem (TOMMERDAHL, 2008, p.10).

Dentro da proposta deste modelo, é importante romper as fronteiras dos campos envolvidos para que a pesquisa colaborativa aconteça efetivamente e, usando aqui as ideias de Koizumi $(1999,2004)$, para que isso aconteça é necessário que haja motivação para o trabalho multidisciplinar, ou ainda, que exista uma 'força motriz' que impulsione a pesquisa, resultando em novas organizações e metodologias de pesquisa, consolidando, desta forma, um novo campo de pesquisa da Mente, Cérebro e Educação, voltado para o melhoramento do processo ensinoaprendizagem.

Fischer (2009) concorda que a pesquisa deve ser colaborativa e de mão dupla, porém, defende a inclusão de profissionais da educação, como professores, técnicos educacionais e os próprios alunos, para trabalharem junto com os pesquisadores e formularem questões e métodos de pesquisa, 
de modo que possam conectar pesquisa e educação. Ele também critica a forma atual de avaliação do ensino-aprendizagem, através de testes padronizados nacionais e internacionais:

O que a educação precisa é de avaliações de performances escolares reais que sejam moldadas por pesquisadores, professores e alunos trabalhando juntos para examinar a eficácia de muitos aspectos da aprendizagem e do ensino no contexto das escolas (currículos, arranjos escolares, tipos de sala de aula, etc.) (FISCHER, 2009, p.4).

$\mathrm{Na}$ verdade, Fischer (2009) propõe mudanças significativas para melhorar a infraestrutura dessas pesquisas colaborativas. Por exemplo, o Movimento MBE (Mind, Brain, and Education), que traz a biologia, a ciência cognitiva e o desenvolvimento humano, juntamente com a educação, para criar uma base científica forte para o ensino e o aprendizado, precisa melhorar a sua infraestrutura e, para tal, Fischer (2009, p.12) propõe três mudanças que são:

(a)a criação de escolas de pesquisa para promover pesquisas que se conectam à prática e à política, (b) o estabelecimento de bancos de dados úteis sobre aprendizagem e desenvolvimento, e (c) a invenção de uma nova classe de educador que se especializa em traduzir entre pesquisa e prática e / ou em engenharia de materiais educativos e atividades baseadas em pesquisa.

Segundo Fischer (2009), essas escolas de pesquisas devem funcionar de forma semelhante aos hospitais universitários que prestam serviços à comunidade, mas que servem também para pesquisas que envolvem estudantes de medicina, profissionais da medicina, pesquisadores e pacientes, reunindo, desta forma, pesquisa e prática. O banco de dados seria semelhante ao trabalho que o INEP, do Ministério da Educação realiza no Brasil, porém, os dados devem incluir a forma como o aprendizado e o ensino ocorrem nas salas de aula, na frente de computadores ou em outros ambientes de aprendizado e não apenas dados estatísticos resultados de testes padronizados. Segundo Fischer (2009), essa iniciativa de criação de Engenheiros Educacionais, que nasce do programa de MBE em Harvard e na 
International MBE Society (IMBES), visa aplicar as descobertas da ciência cognitiva e da neurociência ao aprendizado em sala de aula e pode projetar materiais educacionais e atividades baseadas em pesquisas que promovem o aprendizado em softwares educacionais, na televisão infantil ou em playgrounds.

Muita coisa pode ser feita, quando o espírito de colaboração é colocado acima dos interesses individuais. Esperamos que este artigo possa contribuir de forma reflexiva para o futuro das pesquisas colaborativas que visam aproximar Neurociência e Educação e que as descobertas possam efetivamente ser aplicadas em sala de aula de forma segura, ética e eficiente.

\section{CONCLUSÃO}

O que efetivamente surgiu na 'década do cérebro' foi um grande entusiasmo em aplicar resultados da Neurociência na Educação, evidentemente, para obter respostas para um grande número de questionamentos sobre ensino-aprendizagem. Inicialmente, as preocupações se voltavam para os casos de disfunções cognitivas de origem neurológicas e se expandiu para os problemas gerais de ensinoaprendizagem. O fato é que essa 'explosão em busca de ouro' fragmentou a pesquisa em várias frentes, sem comunicação entre os grupos, com metodologias e organizações independentes, e muitas vezes, com aplicações diretas na educação, sem uma comprovação científica necessária. Isto parece estar sendo contido por organizações compostas por alguns países que tomaram a frente das discussões, na tentativa de estabelecer as bases de um novo campo científico. Embora não se tenha chegado a um consenso, quanto ao rótulo deste novo campo, já se conseguiu estabelecer, que o caminho transdisciplinar é o mais indicado para levar a Neurociência ao encontro da Educação. Na verdade, muito ainda precisa ser discutido, para se colocar em prática esse modelo transdisciplinar, pois, existem vários obstáculos no caminho. Por exemplo, um grande desafio para o trabalho colaborativo neste modelo é derrubar os 
muros que cercam as áreas envolvidas. Motivar pesquisadores ao trabalho colaborativo é uma tarefa difícil que precisa ser superada; além disso, criar ambientes de pesquisa colaborativa com a participação direta de técnicos educacionais, professores e alunos necessita de mudanças profundas na política educacional. Quanto tempo, a Medicina levou para convencer a humanidade da necessidade de estudar a anatomia em cadáveres? Hoje, como mostramos neste artigo, médicos, alunos de medicina, pesquisadores, técnicos, enfermeiros e pacientes convivem nos hospitais universitários espalhados pelo Mundo. Não queremos ser pessimistas, até acreditamos que a Educação já está passando por transformações significativas, porém, são iniciativas particulares de algumas áreas científicas. É necessário mais do que isso, é preciso iniciar uma abordagem "multi-inter-disciplinar" nas pesquisas sobre ensino-aprendizagem nas escolas. Acreditamos que os conhecimentos transdisciplinares surgirão naturalmente, desde que haja um espírito de colaboração, que deixe de lado os interesses particulares dos representantes de cada área envolvida na pesquisa. O mais difícil é convencer profissionais de áreas diferentes a trabalharem juntos. Escrever artigos juntos, já seria um bom começo. Por exemplo, o caso do casal Curie, citado neste artigo. Será que eles planejaram esta transdisciplinaridade que levou a um novo campo científico? Ou será que foi o resultado natural da "multi-interdisciplinaridade", desenvolvida com um interesse comum do casal? O momento é de reflexão, de diálogo e, acima de tudo, de colaboração.

\section{REFERÊNCIAS BIBLIOGRÁFICAS}

ANSARI, D. Mind, Brain, and Education: A Discussion of Practical, Conceptual, and Ethicallssues. Handbook of Neuroethics, pp. 1703-1719. 2015

ANSARI, D.; GRABNER, R. H. Neuroeducation - A Critical Overview of An Emerging Field. Neuroethics, $n^{\circ} 5,105-117,2012$.

BRUER, J.T.Education and the brain: A bridge too far. Educational Researcher, n²6, pp. 4-16, 1997.

BROUSSEAU, G. Fundamentose métodos da didáctica da matemática. In: BRUN, J. (org); FIGUEIREDO, M. J.(tradução). Didáctica das matemáticas. Lisboa: instituto Piaget, P. 35 - 113, 1996. 
FISCHER, K. W. Mind, Brain, and Education: Building a Scientific Groundwork for Learning and Teaching. International Mind, Brain, and Education Society and Wiley Periodicals, Inc. Volume 3-Number 1, 2009.

GOLDSTEIN, M. Decade of the Brain. An Agenda for Nineties. In NeurologyFrom Basics to Bedside (Special Issue), 161: 239-241, 1994.

GOSWAMI, U. Neuroscience and education: from research to practice? Nature reviews neuroscience, v. 7, n. 5, p. 406-413, 2006.

GRABNER, R.H.; ANSARI, D. Promises and potential pit falls of a 'cognitive neuroscience of mathematics learning'. ZDM Mathematics Education, $n^{\circ} 42$, pp. 655-660, 2010.

HOWARD-JONES, P. A. Neuroscience and education: myths and messages. Nature Reviews Neuroscience, v. 15, n. 12, p. 817-824, 2014.

KOIZUMI, H. A practical approach to trans-disciplinary studies for the $21 \mathrm{st}$ century. Journal of Seizon and Life Sciences, 9, 5-24, 1999.

KOIZUMI, H. The concept of 'developing the brain': A new natural science for learning and education. Brain \& Development, 26, 434-441, 2004.

SCHWARTZ, M. Mind, Brain and Education: A Decade of Evolution. International Mind, Brain, and Education Society and Wiley Periodicals, Inc. Volume 9 - Number 2, pp. 64-71, 2015.

OECD, 2002. Understanding the brain: Towards a new learning science. Paris: OECD Publishing.

PECHURA, C. M.; MARTIN, J. B. MAPPING THE BRAIN AND ITS FUNCTIONS: INTEGRATING ENABLING TECHNOLOGIES INTO NEUROSCIENCE RESEARCH. Committee on a National Neural Circuitry Database. NATIONAL ACADEMY PRESS. 1991

ROSE, T.; DALEY, S.; ROSE, D. Let the Questions be Y our Guide: MBE as InterdiciplinaryScience. Mind, Brain, and Education, 5(4), 153-162, 2011.

RATO, J.; CALDAS, A. Neurociências e educação: Realidade ou ficção? Actas do VII Simpósio Nacional de Investigação em Psicologia Universidade do Minho, Portugal, 4 a 6 de Fevereiro de 2010, 2010.

SAMUELS, B. M. Can the differences between education and neuroscience be overcome by mind, brain, and education? Mind, Brain, and Education, 3, $44-54,2009$.

TANDON, P. The Decade of The Brain: A Brief Review. Neurology India., 48:199$207,2000$. 
TOMMERDAHL, J. Education and the neurosciences: where are we? SENCO Update, 95, 8-10, 2008.

TOKUHAMA-ESPINOSA, T. N. The Scientifically Substantiated Art of Teaching: A Study in the Development of Standards in the New Academic Field of Neuroeducation (Mind, Brain, and Education Science). A Dissertation Presented in Partial Fulfillment of the Requirements for the Degree of Doctor of Philosophy. Capella University, 2008.

VANINSKY, A. Educational Neuroscience, Educational Psychology, and Classroom Pedagogy as a System. American Journal of Educational Research, Vol. 5, No. 4, 384-391, 2017.

Recebido em: 25 de janeiro de 2019. Aprovado em: 13 de maio de 2019. 